\title{
Real-time fiber selection using the Wii remote
}

\author{
Jan Klein ${ }^{a}$, Mike Scholl ${ }^{a}$, Alexander Köhn ${ }^{a}$, and Horst K. Hahn ${ }^{a}$ \\ ${ }^{a}$ Fraunhofer MEVIS, Center for Medical Image Computing, Bremen, Germany
}

\begin{abstract}
In the last few years, fiber tracking tools have become popular in clinical contexts, e.g., for pre- and intraoperative neurosurgical planning. The efficient, intuitive, and reproducible selection of fiber bundles still constitutes one of the main issues. In this paper, we present a framework for a real-time selection of axonal fiber bundles using a Wii remote control, a wireless controller for Nintendo's gaming console. It enables the user to select fiber bundles without any other input devices. To achieve a smooth interaction, we propose a novel spacepartitioning data structure for efficient 3D range queries in a data set consisting of precomputed fibers. The data structure which is adapted to the special geometry of fiber tracts allows for queries that are many times faster compared with previous state-of-the-art approaches. In order to extract reliably fibers for further processing, e.g., for quantification purposes or comparisons with preoperatively tracked fibers, we developed an expectationmaximization clustering algorithm that can refine the range queries. Our initial experiments have shown that white matter fiber bundles can be reliably selected within a few seconds by the Wii, which has been placed in a sterile plastic bag to simulate usage under surgical conditions.
\end{abstract}

Keywords: brain, neurosurgical procedures, intraoperative procedures, visualization, diffusion tensor imaging

\section{INTRODUCTION}

Over the last years, diffusion imaging techniques like DTI, ${ }^{1,2}$ DSI or Q-Ball ${ }^{3}$ received increasing attention, especially in the neuroimaging, neurological, and neurosurgical ${ }^{4}$ community. An explicit geometrical reconstruction of major white matter tracts has become available by fiber tracking (FT) based on diffusion-weighted images. The goal of virtually all FT algorithms is to compute results which are analogous to what the physicians or radiologists are expecting and an extensive amount of research has therefore been focussed on this reconstruction. ${ }^{1-3,5}$ Current research aims at improving the reconstruction process by GPU implementations ${ }^{6-8}$ which are $^{-1}$ able to track about 50,000 fibers within one second. ${ }^{8}$

In practice, the interesting structures are not individual fibers, which in any case are impossible to reconstruct since the resolution of diffusion-weighted images is much lower than the diameter of the individual fibers. Instead, the interesting structures are anatomical meaningful bundles that fibers form. These bundles may be used for statistical analysis, monitoring disease progression, or planning of a neurosurgical approach.

Fiber clustering techniques have mainly been developed for selecting anatomically meaningful bundles in order to perform statistical measurements. ${ }^{9,10}$ They are relatively inflexible for selecting fibers within a certain $3 \mathrm{D}$ range. A more promising way to explore these bundles is through the use of fiber selection tools. These approaches either determine the fibers on-the-fly by computing them from user-defined seed points or the fibers may be selected from a preprocessed whole brain fiber tracking result. Algorithms of the first category are often superior with respect to real-time and memory requirements as only few fibers have to be tracked. The selection from a whole brain fiber tracking result, however, is often superior in the quality with respect to completeness and uncertainty of the results due to the non-bijetivity of FT, see Fig. 1 and Fig. 2. New approaches ${ }^{11,12}$ simplify the selection process by utilizing $2 \mathrm{D}$ representations of fiber tracts.

Besides these two strategies, the modality of the selection is an important research field. Desired fiber bundles can often be selected by using Boolean expressions involving one or more user-defined ROIs (regions of interest). ${ }^{13,14}$ New input techniques attempt to circumvent the problem that scientists would like to position and resize ROIs in 3D with commodity input devices such as mice and pens that only provide two degrees of

Further author information: (Send correspondence to Jan Klein)

Jan Klein: E-mail: jan.klein@mevis.fraunhofer.de, Telephone: 494212188902 

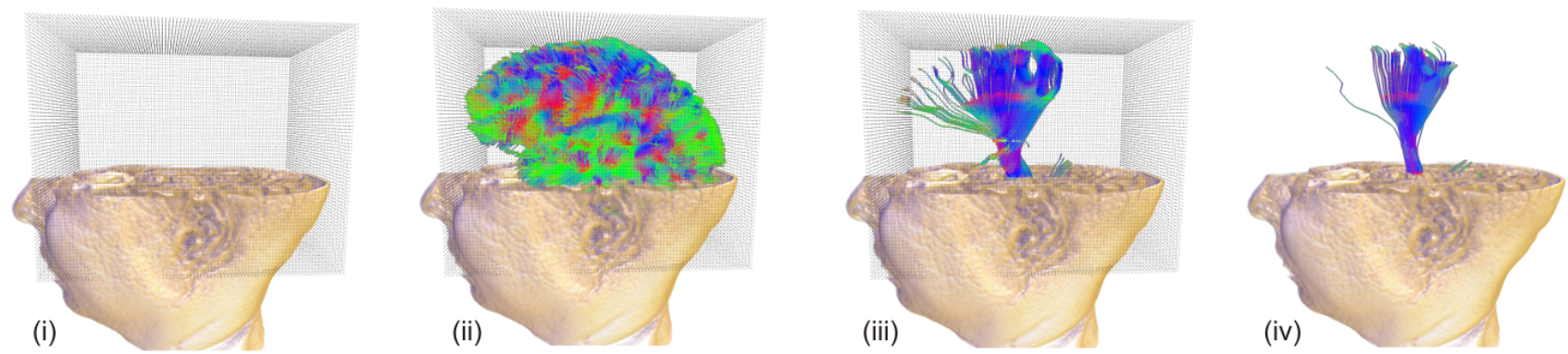

Figure 1. Seed-ROI-based fiber tracking versus whole brain fiber tracking. (i) Seed points used for a whole brain fiber tracking. (ii) Result of a whole brain fiber tracking. (iii) Fibers from (ii) are shown which pass a user-defined ROI. (iv) Fibers are missing, especially at the boarders of the tracked bundle, if using a seed-ROI approach.

freedom. ${ }^{15,16}$ Such methods augment the selection process with a set of marking operations that are both natural and expressive. However, such methods still use 2D input devices for selection.

In this paper, we present a framework that allows real-time fiber selection by utilizing a Wii remote controller (abbreviated as Wii in the following). The Wii is the primary controller for Nintendo's Wii gaming console. A main feature of the Wii is its motion sensing capability, which allows the user to interact with and manipulate items on screen via movement and pointing through the use of accelerometer and optical sensor technology. The intended use of the Wii is to provide a robust, efficient, and easy 3D interaction device to select fiber bundles in intraoperative situations without leaving the operating table. The intraoperative selection is practical in situations in which a brain shift does not occur, e.g, for minimally invasive stereotactic operations, or those in which intraoperative image acquisition is available to correct the brain shift. An elaborate manual selection of fiber bundles is not possible due to the restricted amount of time available and the sterile environment in which the use of a mouse is not feasible. The Wii is widespread and cheap and may easily be placed in a sterile plastic bag. The Wii permits controlling a visualization system that can be stationed several meters away from the situs.

Our goal is to select the bundles from a whole-brain fiber tracking in which fibers have been precomputed for all voxels in a brain with respect to a specific resolution of the seed grid. In contrast to simple ROI-based seeding algorithms, the selection from a whole-brain fiber tracking is often superior in quality with respect to completeness and uncertainty of the results due to the non-bijetivity of fiber tracking. Thus, we have to develop an efficient solution that determines the desired fibers from a large set of preprocessed fibers within the span of a few milliseconds, guaranteeing a smooth interaction with the Wii. To enhance the fiber selection, we developed an expectation-maximization (EM) clustering technique that may refine the fiber selection.

\subsection{Contributions}

The new contributions of this paper can be summarized as follows:

- A novel space-partitioning data structure for efficient range queries is adapted to the special geometry of streamlines. The data structure allows for ranges queries that are three times faster than previous state-of-the-art techniques. The data structure is easy to implement and integrate into other fiber-tracking tools.

- The adaption of a Wii remote controller as an input device for the selection process enables the user to interactively select fiber bundles without any other input device. This makes the Wii very useful for intraoperative situations in which no brain shift appears, image data can be intraoperatively acquired, or a brain shift correction is available. Our tests have shown that the Wii can be placed in a sterile plastic bag without decreasing the selection precision. To the best of our knowledge, fiber bundle selection has not been previously accomplished using a Wii.

- The developed expectation-maximization clustering approach can refine the range queries. Thus, fibers could be reliably extracted and further processed, e.g., for quantification purposes or comparisons with preoperatively tracked fibers. 

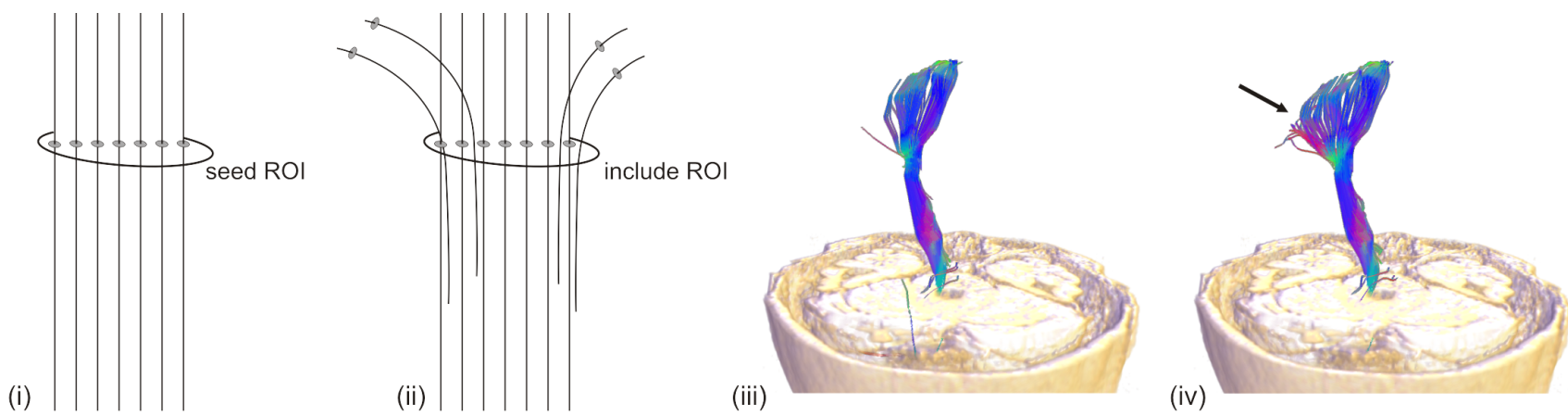

Figure 2. (i) Seed-ROI-based fiber tracking. Only seed points within the ROI are used for fiber tracking. (ii) Seed point which are distributed over the whole brain are used for the whole brain fiber tracking. Afterwards, all fibers passing a ROI can be shown. (iii) Seed-ROI-based fiber tracking. (iv) Whole brain fiber tracking.

The paper is organized as follows. We explain our novel data structure and hierarchy traversal in Section 2. Furthermore, the adaption of the Wii remote including the refinement by an EM clustering is demonstrated. Results are presented afterwards (Section 3) and finally, we will discuss our work and give some ideas for future work (Section 4).

\section{METHODS}

For efficient 3D range queries, in other words, computing fibers lying within a certain 3D range described by some kind of bounding volume, it is impracticable to test all fibers from a whole brain fiber tracking against their intersection with the selection object because each fiber may consist of 50 to 100 fiber segments, and a whole brain fiber tracking may consist of more than 100,000 fibers. Instead, space-partitioning data structures ${ }^{14}$ are used to test only small parts of fibers against the selection object.

The total cost of a 3D range query may be roughly estimated as $T=C_{v} N_{v}+C_{f} N_{f}$ where $N_{v}$ denotes the number of bounding volume pair overlap tests, $N_{f}$ the number of fiber segments tested for intersection, and $C_{v}, C_{f}$ the individual intersection costs. Thus, we can minimize the number of bounding volumes by tightening them, or we can minimize the costs for intersection tests by simplifying the bounding volumes, or we can try to find a good compromise between both. In literature, especially in the context of collision detection, much effort has been spent on this problem and until now, no general solution has been shown that is efficient for a broad range of applications.

As optimizing the cost function $T$ for the special geometry of fiber segments has not been thoroughly researched, we propose a novel data structure, called fiber tree. Based on, ${ }^{14}$ we decided to use a $\mathrm{kD}$-tree as starting basis as well, but our new data structure (fiber tree) has been highly optimized with respect to its construction process. As we enclose the fibers by hierarchically organized bounding volumes (BVs) in a hybrid way (oriented bounding boxes (OBBs) enclosing the fibers and a $\mathrm{kD}$-tree enclosing the OBBs), the number of visited nodes during a $3 \mathrm{D}$ range query is minimized.

Furthermore, if we allow for small, controllable approximation errors, the worst-case running time can be determined. If using a $\mathrm{kD}$-tree combined with a straight approximation as proposed in, ${ }^{14}$ the error cannot be handled and a polylogarithmic running time cannot be guaranteed in the worst case.

\subsection{Selection of separating planes}

It has already been shown in the context of computational geometry that $\mathrm{kD}$-trees are more efficient with respect to range queries when cutting the single BVs on the longest side. ${ }^{17}$ Thus, such $\mathrm{kD}$-trees should be preferred to simple kD-trees. Furthermore, we propose to exploit the geometrical fiber information so that the BVs do not have to be simply cut at the center of the longest side. More precisely, we perform a spectral fiber clustering ${ }^{18}$ of the available fibers within each node and use the cluster information in order to determine a separating plane which aims to cut as few clusters as possible, see Fig. 3. Thereby, we start with the longest side, but if no satisfactory result can be determined, we try the other two planes. In cases in which too many fibers of different 


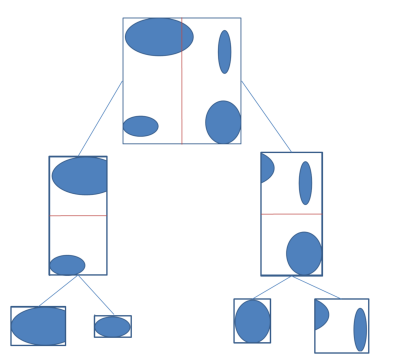

(i)

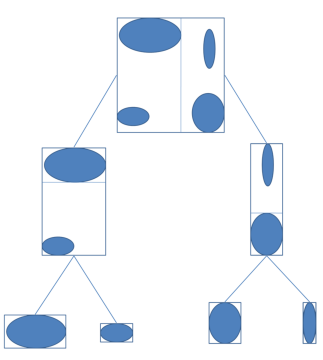

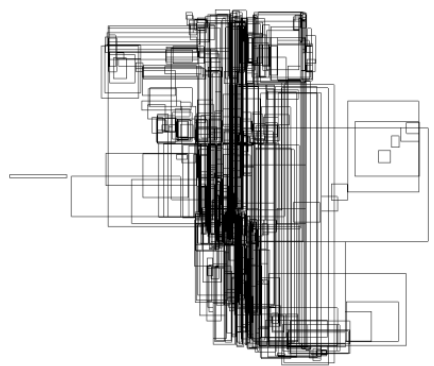

(ii)

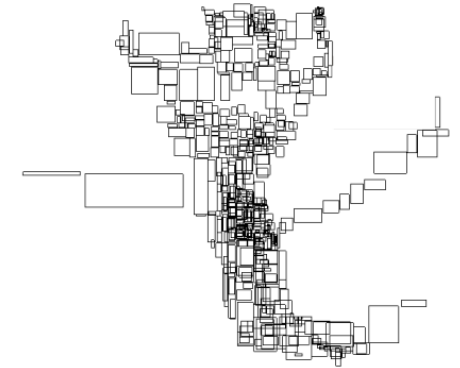

Figure 3. We examined different strategies to compute the separating planes during the tree construction. (i) Left: standard construction using the center for separating a bounding volume. Right: separating planes computed by spectral fiber clustering. (ii) BVs enclosing fibers of the pyramidal tract. Left: kD-tree using alternating separating planes. Right: fiber tree where separating planes are determined by spectral fiber clustering. Note that in both cases only bounding volumes of the same depth are shown.

clusters are cut or if the BVs are cut very unequally, we choose the median and discard the information obtained by the spectral clustering. Fiber segments that are intersected by a separating plane have to be inserted into both child nodes.

\subsection{Enclosing the fibers}

To minimize the number $N_{f}$ under the condition to keep $C_{f}$ low, we propose to enclose the fibers by oriented bounding boxes (OBBs) instead of inserting the fiber segments directly into the fiber tree, see Fig. 4. Each fiber is partitioned into subsets depending on the local curvature, see Fig. 4 (right). Afterwards, an OBB is computed by a principal component analysis (PCA) for each subset.

\subsection{Hierarchy traversal}

Given a 3D selection object, a range query may easily be performed by traversing the tree into those parts where the object intersects the corresponding BVs. Additionally, at intersecting leaf nodes, the OBBs, enclosing fiber parts must be tested. This can be carried out efficiently, as described in. ${ }^{19}$ Only in the case of an intersection must the corresponding single fiber segments be tested. Optionally, our framework supports approximate range searching $^{20}$ in which single fiber segments may be undetected within a small, definable $\epsilon$-border around the BVs of the fiber tree. The advantage of such an approach is not only an improved running time, but the worst-case performance of our approach can be estimated by $O\left(1 / \epsilon^{2} \log ^{2} n\right)$ where $n$ denotes the number of all fiber points.

\subsection{Controlling a 3D ROI using the Wii}

Recent APIs ${ }^{21}$ offer a data exchange between the Wii and an arbitrary operating system using the open Bluetooth standard for data transfer. Thus, the Wii runs independently of the gaming console and can be used in conventional applications. In order to take advantage of the Wii's capabilities, we integrated an open-source Bluetooth $\mathrm{API}^{21}$ for the Wii into our software platform, which is available for Windows and Mac OS. To utilize the optical sensor technology of the Wii, a sensor bar has to be installed near the interaction screen. The sensor bar features ten infrared LEDs, five of which are arranged at each end of the bar. Thus, the Wii can locate the LEDs (i.e., the relative position of the interaction screen) by means of an integrated infrared camera. Each of the five infrared rays are bundled within a single point of a $2 \mathrm{D}$ coordinate system of the camera. Thus, two $2 \mathrm{D}$ points can be read out, which change if the Wii is moved. From these two points, a "left-right" as well as an "up-down" movement of the selection object can easily be computed within the local coordinate system. Furthermore, this also allows rotating the skull or the selection object. For this purpose, only the mode has to be changed from "moving selection object" to "rotating skull" or "rotating selection object" by simply clicking a predefined button of the Wii. The depth, i.e., a "front-back" movement, could be achieved by evaluating the distance between the two projected points. However, the resolution is too coarse for a fine-tuned movement. Instead, we propose to use two available buttons of the Wii, e.g., the key-up and the key-down buttons of the directional pad (D-pad). Note that during motion or rotation of the selection object or skull, the B-button at 

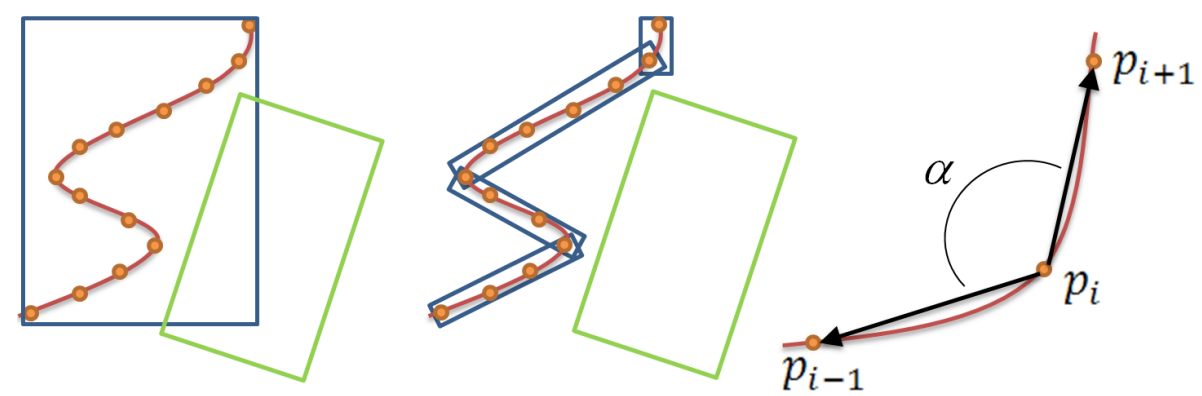

Figure 4. Instead of enclosing the fibers by an axis-aligned bounding box (left), we enclose parts of them by tighter OBBs (center). As a consequence, the selection object (green box) has less often to be tested against the fiber segments. Right: If the angle $\alpha$ between two consecutive fiber segments is too large, a new OBB is generated.

the back of the Wii has to be pressed in our implementation. Otherwise, small movements of the Wii and thus of the selection object would be permanently performed. For operating room use, the Wii can be enclosed in a sterile plastic bag. In order to enable precise user interaction, the location of the screen, its size and the distance between user and screen must be adequate. Assuming a 19" display, we found that a distance of $0.5 \mathrm{~m}-3.0 \mathrm{~m}$ between user and screen is adequate for the intended interaction tasks.

\subsection{Refining the selection process}

To enhance the selection process, we developed a technique that can optionally be used. It allows for the classification of fiber tissue and background within a certain volume of interest. After the classification, only streamlines inside fibrous tissue are displayed, see Fig. 5. In contrast to a simple thresholding of the fractional anisotropy (FA), our method does not discard fibers in which the FA may be partially low. More precisely, inside the selection object, we propose a probabilistic mixture model that includes the two classes fiber (f) and background (b). In addition, a partial volume class, which is a mixture of (f) and (b), is added to the model. This is intended to make results independent of the resolution of the data. We use the diffusion tensor in each voxel inside the selection object for the classification of the data which is based on an expectation-maximization algorithm.

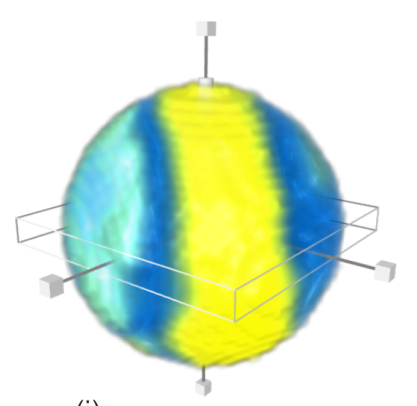

(i)

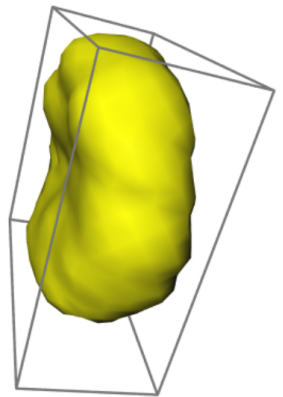

(ii)
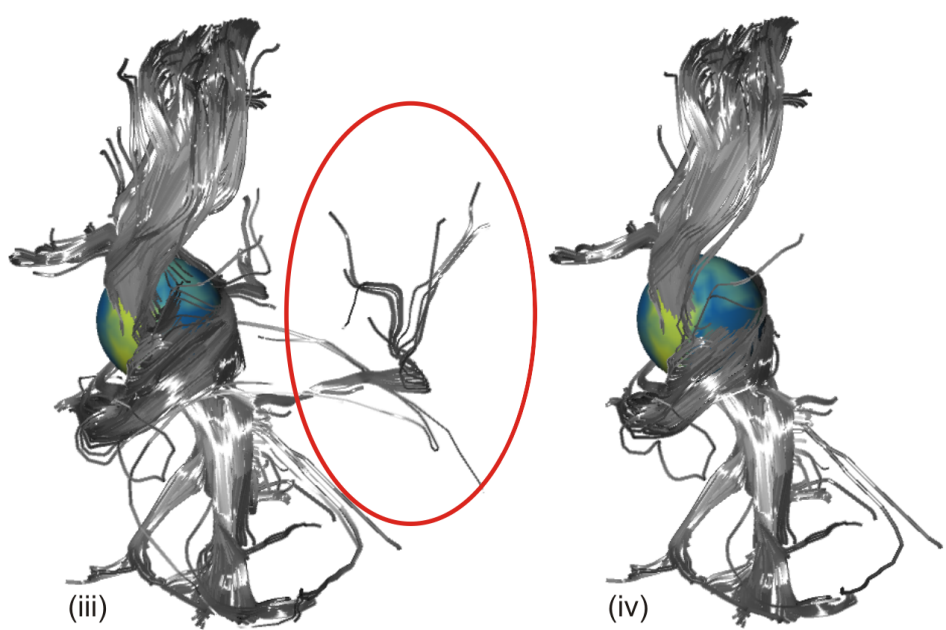

Figure 5. The selection of fibers can be refined by our EM clustering. (i): the volume of interest has been classified into fibrous and non-fibrous structure as well as into partial volume. (ii): OBB enclosing the fibrous structure. (iii) without EM clustering (iv) with EM clustering, fibers not belonging to pyramidal tract are not displayed. 

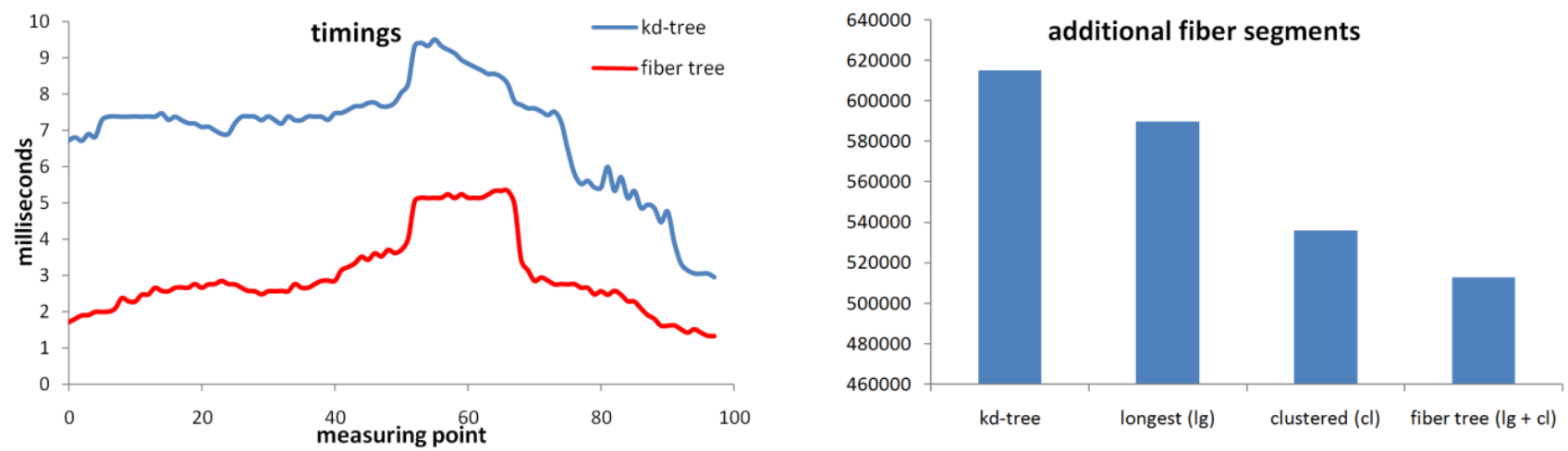

Figure 6. Left: a comparison of the timings for a fixed path through the 3D scene. On average, our fiber tree allows for search queries which are three times faster compared to a kD-tree. Right: additional fiber segments depending on the used data structure.

\section{RESULTS}

We have implemented all proposed algorithms and data structures in $\mathrm{C}++$. The results were obtained using a PC with a $3.0 \mathrm{GHz}$ Intel Core2 Quad Extreme Q6850 and a GeForce 8800 Ultra graphics card. For timing the performance and measuring parameters like the number of intersection tests, fiber tracts were computed from DTI data acquired at a 1.5T Siemens scanner using a deflection-based fiber tracking algorithm which has been implemented on the GPU (the shaders are written in GLSL). A whole brain fiber tracking of 40,000 fiber tracts can be performed within one second. The construction of our fiber tree can be performed within a few seconds and can be saved and read from disk. Fig. 6 (right) indicates the number of additional fiber segments that are inserted due to intersections with separating planes. A screenshot of our software in which fiber tracts are interactively displayed in the vicinity of a large tumor is given in Fig. 8. There, the fibers are selected and visualized in real-time by moving the Wii. Timings of 3D search queries along a predefined path consisting of 100 steps are given in Fig. 6 (left). The number of traversed nodes and the number of intersection tests are shown in Fig. 7.

Our initial experiments have shown that white matter fiber bundles can be selected within a few seconds by the Wii. For this task, we defined 5 bundles (pyramidal tracts, cingulum, superior longitudinal fasciculus, visual pathways, fornix) to be selected by the user. It has been shown that the required time is nearly the same as when manually drawn ROIs are used for the selection process.
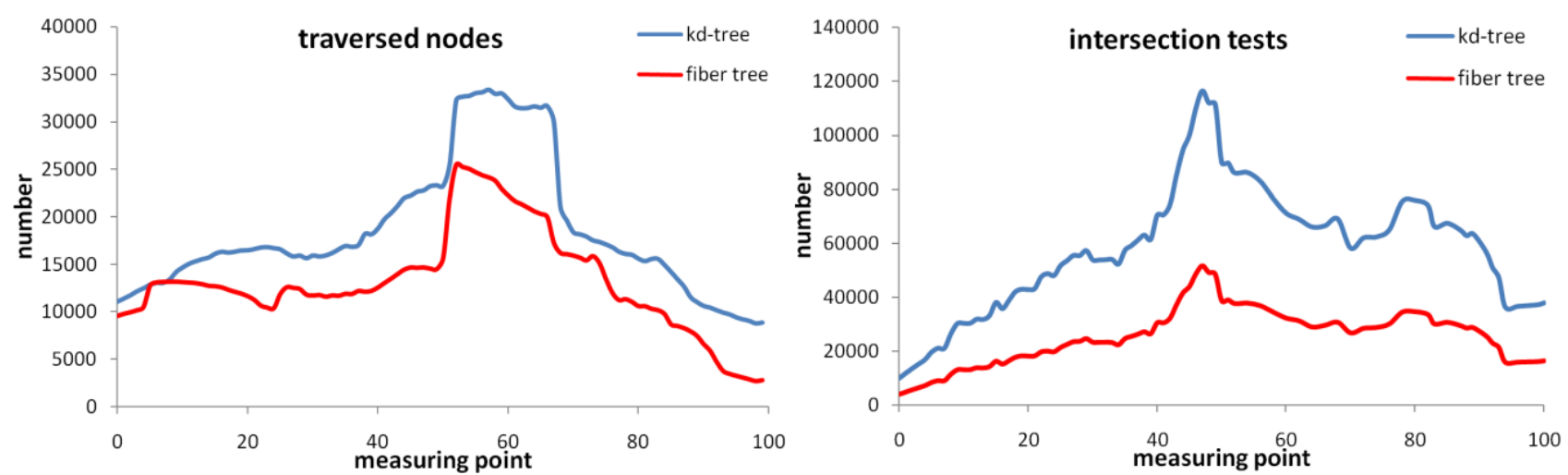

Figure 7. Compared to a $\mathrm{kD}$-tree, the number of traversed nodes and the number of intersection tests is lower if using our fiber tree. 


\section{CONCLUSIONS AND FUTURE WORK}

Our results have shown that our fiber tree performs well for interactively selecting fibers. To the best of our knowledge, this has not been previously accomplished using a Wii remote controller. The timings show that our fiber tree allows for search queries that are about three times faster than a $\mathrm{kD}$-tree. This is due to a reduced number of traversed nodes, a reduced number of additionally inserted fiber segments, and a reduced number of intersection tests. The number of traversed nodes has been reduced by a better fitting of the geometry by our clustering and construction strategy (longest side). The number of additionally inserted fiber segments could be reduced because the clustering strategy aims to separate the fiber tracts into subvolumes under the condition to intersect as few segments as possible. As a consequence, the intersection tests are reduced. The enclosing of fiber segments by OBBs leads to a further reduction of intersection tests. The selection using a Wii has shown to be very comfortable. The "front-back" movement that we initially implemented by evaluating the distance between the two projected points on the Wii has shown to be too coarse. Independent of this, some users did not like to move the Wii towards and away from the monitor. Our solution to replace the "front-back" movement by simply pressing a Wii button has shown to be an acceptable solution. In future work, it should be examined whether two Wiis (prototypically bound together) can improve on this.

The refinement of the fibers by the proposed EM clustering is useful if the selection object contains both fibrous and background tissues. Using such a method, fibers could be extracted reliably and further processed, e.g., for quantification purposes or comparisons with preoperatively tracked fibers. However, if no background is included, the EM cluster-ing produces no advantage. Due to the time-consuming clustering step that lasts one or two seconds, the refinement can only be performed if the Wii is not turned by the user. In future work, it would be interesting to do user studies in order to examine whether our proposed framework could also be utilized for demonstration or educational purposes.

\section{REFERENCES}

1. S. Mori, B. Crain, V. Chacko, and P. van Zijl, "Three-dimensional tracking of axonal projections in the brain by magnetic resonance imaging," Ann Neurol. 45(2), pp. 265-269, 1999.

2. P. Basser, S. Pajevic, C. Pierpaoli, J. Duda, and A. Aldroubi, "In vivo fiber tractography using dt-mri data," Magn Reson Med. 44(4), pp. 625-632, 2000.

3. D. Tuch, "Q-ball imaging," Magnetic Resonance in Medicine 52, pp. 1358-1372, 2004.

4. C. Nimsky, O. Ganslandt, P. Hastreiter, R. Wang, T. Benner, A. G. Sorensen, and R. Fahlbusch, "Preoperative and intraoperative diffusion tensor imaging-based fiber tracking in glioma surgery," Neurosurgery 56(1), pp. 130-138, 2005.

5. O. Friman, G. Farnebäck, and C.-F. Westin, "A Bayesian approach for stochastic white matter tractography," IEEE Trans. Medical Imaging 25(8), pp. 965-978, 2006.

6. T. McGraw and M. Nadar, "Stochastic dt-mri connectivity mapping on the gpu," IEEE Trans. Vis. Comput. Graph. 13(6), pp. 1504-1511, 2007.

7. P. Kondratieva, J. Kruger, and R. Westermann, "The application of gpu particle tracing to diffusion tensor field visualization," in Proc. of IEEE Vis 2005, pp. 73-78, 2005.

8. A. Köhn, J. Klein, F. Weiler, and H.-O. Peitgen, "A gpu-based fiber tracking framework using geometry shaders," Medical Imaging 2009: Visualization, Image-Guided Procedures, and Modeling 7261(1), p. 72611J, SPIE, 2009.

9. M. Maddah, A. Mewes, S. Haker, W. E. L. Grimson, and S. Warfield, "Automated atlas-based clustering of white matter fiber tracts from DTMRI," in MICCAI'05, pp. 188-195, 2005.

10. M. Caan, L. Vliet, C. Majoie, E. Aukema, K. Grimbergen, and F. Vos, "Spatial consistency in 3d tract-based clustering statistics," in MICCAI'08, pp. 535-542, 2008.

11. W. Chen, Z. Ding, S. Zhang, A. MacKay-Brandt, S. Correia, H. Qu, J. A. Crow, D. F. Tate, Z. Yan, and Q. Peng, "A novel interface for interactive exploration of dti fibers," IEEE Transactions on Visualization and Computer Graphics 15(6), pp. 1433-1440, 2009.

12. R. Jianu, C. Demiralp, and D. Laidlaw, "Exploring 3d dti fiber tracts with linked 2d representations," IEEE Transactions on Visualization and Computer Graphics 15(6), pp. 1449-1456, 2009. 


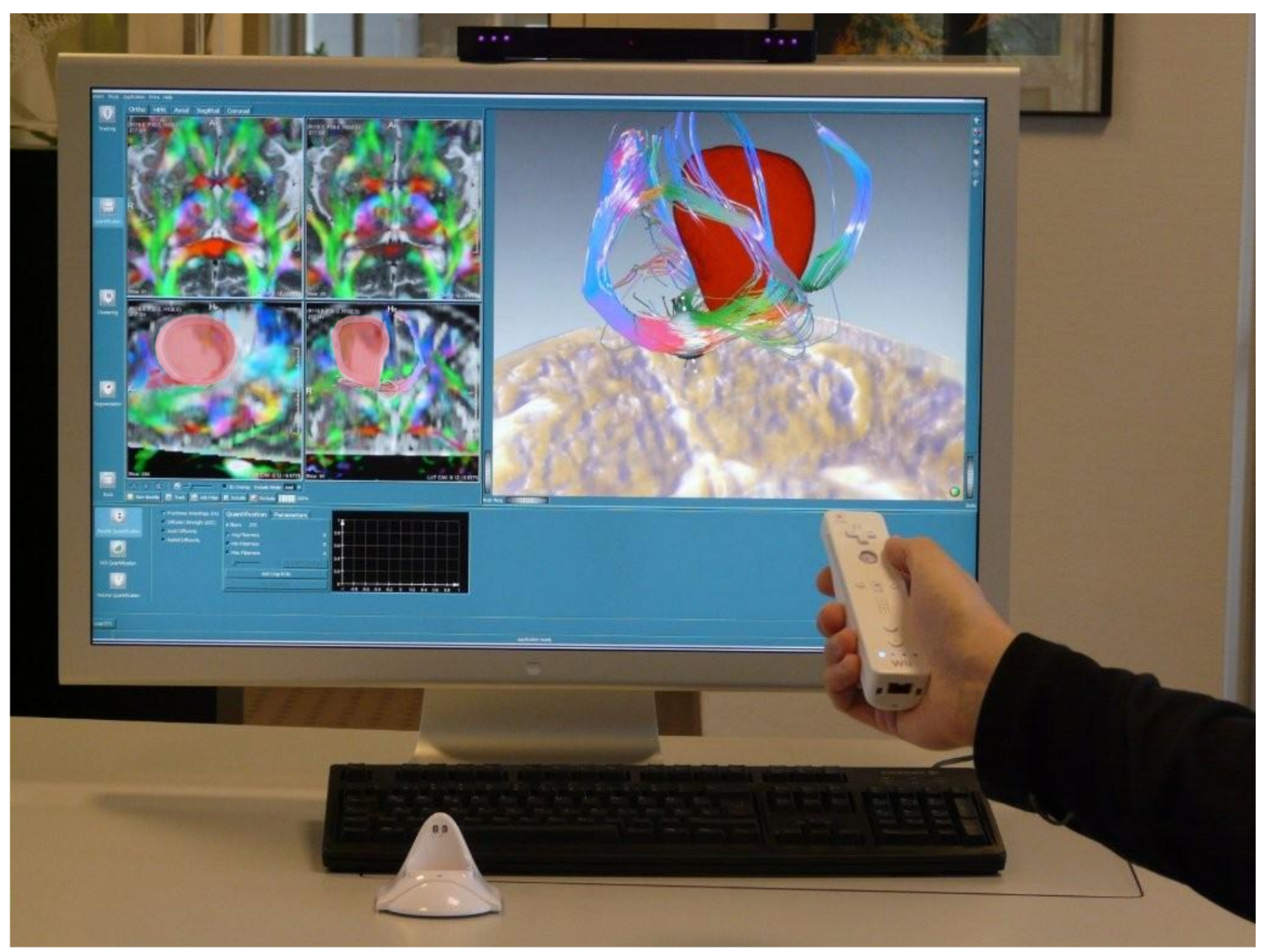

Figure 8. Fibers in the vicinity of a tumor are selected with the help of a Wii remote controller.

13. A. Sherbondy, D. Akers, R. Mackenzie, R. Dougherty, and B. Wandell, "Exploring connectivity of the brain's white matter with dynamic queries," IEEE Trans. Vis. Comput. Graph. 11(4), pp. 419-430, 2005.

14. J. Blaas, C. P. Botha, B. Peters, F. Vos, and F. H. Post, "Fast and reproducible fiber bundle selection in dti visualization," in IEEE Visualization, pp. 59-64, 2005.

15. D. Akers, "Cinch: a cooperatively designed marking interface for $3 \mathrm{~d}$ pathway selection," in Proc. of the 19th annual ACM symposium on User interface software and technology (UIST'06), pp. 33-42, 2006.

16. T. Igarashi, S. Matsuoka, and H. Tanaka, "Teddy: a sketching interface for 3d freeform design," in SIGGRAPH'99, pp. 409-416, 1999.

17. M. Dickerson, C. A. Duncan, and M. T. Goodrich, "K-d trees are better when cut on the longest side," in Proc. of the 8th Annual European Symposium on Algorithms (ESA'00), pp. 179-190, 2000.

18. J. Klein, P. Bittihn, P. Ledochowitsch, H. K. Hahn, O. Konrad, J. Rexilius, and H.-O. Peitgen, "Gridbased spectral fiber clustering," Medical Imaging 200\%: Visualization and Image-Guided Procedures 6509(1), p. 65091E, SPIE, 2007.

19. S. Gottschalk, M. C. Lin, and D. Manocha, "OBBTree: a hierarchical structure for rapid interference detection," in SIGGRAPH'96, pp. 171-180, 1996.

20. S. Arya and D. M. Mount, "Approximate range searching," in Proc. of 11th Annu. ACM Sympos. Comput. Geom, pp. 172-181, 1995.

21. Wii Brew, "http://wiibrew.org," 2009. 